\title{
REVIEW
}

\section{Left bundle branch pacing: the new kid on the block}

\author{
Andra Gurgu', Dragos Cozma', Mihail G. Chelu²
}

\begin{abstract}
Right ventricular pacing is has deletorius effects due to left ventricular dysynchrony and remodelling and may result in heart failure. Over the last decade, His bundle pacing has emerged as the most physiologic form pacing. However, it has limitations, such as higher capture thresholds, lower $\mathrm{R}$ wave amplitudes, atrial oversensing, and increased risk for lead revisions from late threshold increase with subsequent premature battery depletion, which has prevented a wider adoption of this technique in routine clinical practice. Left bundle branch pacing has been developed as an alternative physiologic pacing strategy that overcomes most of His bundle pacing limitations. This article summarizes the current status of left bundle branch pacing.
\end{abstract}

Keywords: His bundle pacing, left bundle branch pacing, cardiac resynchronization, therapy heart failure.

\section{INTRODUCTION}

Left bundle branch block (LBBB) has been shown to increase morbidity and mortality in heart failure patients due to ventricular dyssynchrony. The marker of ventricular dyssynchrony is the wide QRS duration (QRSd) that sugest nonsimultaneous activation of the ventricular walls. As a result of prolonged ventricular conduction time and dyssynchronous activation of the ventricles, systolic dysfunction may occur in patients with LBBB'.

Using (HBP) the native conduction system, His bundle pacing has been proved to be the most physiologic form of ventricular pacing, than can correct wide QRSd, ensure the ventricular electrical activation synchrony and improves the clinical outcomes in heart failure patients ${ }^{1-3}$. However the technique is limited by difficult implantation, long fluoroscopic exposure time, high capture threshold and fails to provide adequate pacing in patients with infrahisian block or proximal left bundle branch block ${ }^{4}$.

By providing a stable and low capture threshold, left bundle branch pacing has emerged to be a potential altenative to His bundle pacing in heart failure patients with left bundle branch block and furthermore an attractive option for pacing in patients with infranodal conduction disease ${ }^{5-7}$.

\footnotetext{
' Department of Cardiology, Institute of Cardiovascular Diseases,

Timisoara, Romania

${ }^{2}$ Division of Cardiology, Baylor College of Medicine, Houston, TX, USA
}

\section{HOW TO PERFORM LBBP}

Left bundle branch pacing was first described Huang and colleagues in 2017. After unsuccessful attempt to implant a left ventricular lead and high pacing thresholds with His bundle pacing they were able to correct left bundle branch block with pacing in the left bundle area in a patient with heart failure, a left ventricular ejection fraction of $32 \%$, NYHA class IV, and left bundle branch block with a QRSd of $180 \mathrm{~ms}^{5}$. Patient experienced great improvement in all heart failure parameters: LVEF increased to $62 \%$, the LV end-diastolic volume decreased from $76 \mathrm{~mm}$ to $42 \mathrm{~mm}$, serum Btype natriuretic from $577 \mathrm{pg} / \mathrm{mL}$ to $20 \mathrm{pg} / \mathrm{mL}$, NYHA class from IV to I and there were no hospitalization for heart failure ${ }^{5}$.

Preprocedural echocardiography is recommended to assess the thickness of the basal interventricular septum and the presence of septal scar ${ }^{8}$. Dilatation of the right atrium and/or right ventricle, bulging of interventricular septum into the right ventricle, LV hypertrophy, septal scar, or significant tricuspid regurgitation can impact procedural succes ${ }^{8}$. In patients with $\mathrm{LBBB}$, it is recommended to have ventricular backup pacing because complete AV block may occur due to RBB injury ${ }^{8}$.

\section{Contact address:}

Mihail G. Chelu, MD, PhD, FHRS

Division of Cardiology, Baylor College of Medicine, 7200

Cambridge Suite A6.137, MS: BCM62I, Houston, TX 77030.

E-mail: Mihail.Chelu@bcm.edu 
LBBP is typically performed using a Select Secure 3830 pacing lead (Medtronic Inc, Minneapolis, MN) and a catether-delivering sheath $(\mathrm{C} 3 \mathrm{I} 5 \mathrm{H}$ is or Select site $\mathrm{C} 304 \mathrm{His}$ ) (Figure I) via left subclavian or left axillary vein access ${ }^{8}$. Intracardiac and 12-lead electrocardiograms are continuously recorded and displayed on an electrophysiological recording system. The catheter and pacing lead are placed into the His bundle area in $30^{\circ}$ right anterior oblique fluoroscopic view and the His bundle potential is recorded ${ }^{8,9}$. After noting the His bundle location, the sheath/pacing lead is advanced $1.5-2 \mathrm{~cm}$ apically, ${ }^{8,9}$. Pacing showing a "W" with the notch closer to the nadir in lead VI may indicate an ideal postion ${ }^{8}$. The sheath is manipulated, usually by rotating it counterclockwise, until the pacing asssumes a position perpendicular to the interventricular septum (Figure 2B, C). The lead is the gradually screwed in with a clockwise rotation. During lead advancement, paced QRS morphology and pacing impedance are monitored ${ }^{8-10}$.

As the lead is advanced, the paced QRS morphology changes from a LBB pattern to a right bundle branch block (RBBB) pattern in lead VI (qR or rsR') ". Initially the impedance increases gradually before it drops by about $100 \Omega$ when the lead tip reaches the LBB. Other means to confirm sufficient depth of the lead and proximity to the LBBB include: unipolar ring pacing capture the septum, fulcrum sign, estimation of depth by injecting contrast through the sheath and delineating the septum, and recording of LBB potential when present. ${ }^{8-10}$ Lead depth can also be assessed by echocardiogram.

The paced QRS morphology could be influenced by the LBB pacing site, existing bundle disease or selective or nonselective LBBP capture ${ }^{8-10}$.

The following criteria for LBB capture have been proposed: paced RBB pattern; recording of LBB potential; evidence of direct LBB capture; short $(\leq 60$ $90 \mathrm{msec}$ ) and constant at high and low output left ventricular activation time (LVAT) defined as interval between pacing stimulus to peak of $R$ wave in lead V5/ V6; determination of selective vs non-selective LBB pacing.

With selective LBB capture, the paced QRS morphology usually has a rSR pattern in VI and there is an isolectric interval between the pacing stimulus and the onset of QRS complex". Nonselective LBBP is defined by a paced QRS with a QR pattern in VI and no isoelectric interval between the pacing spike and the QRS complex".

Another method to differentiate LBB capture vs LV septal myocardial capture relies on the differen-
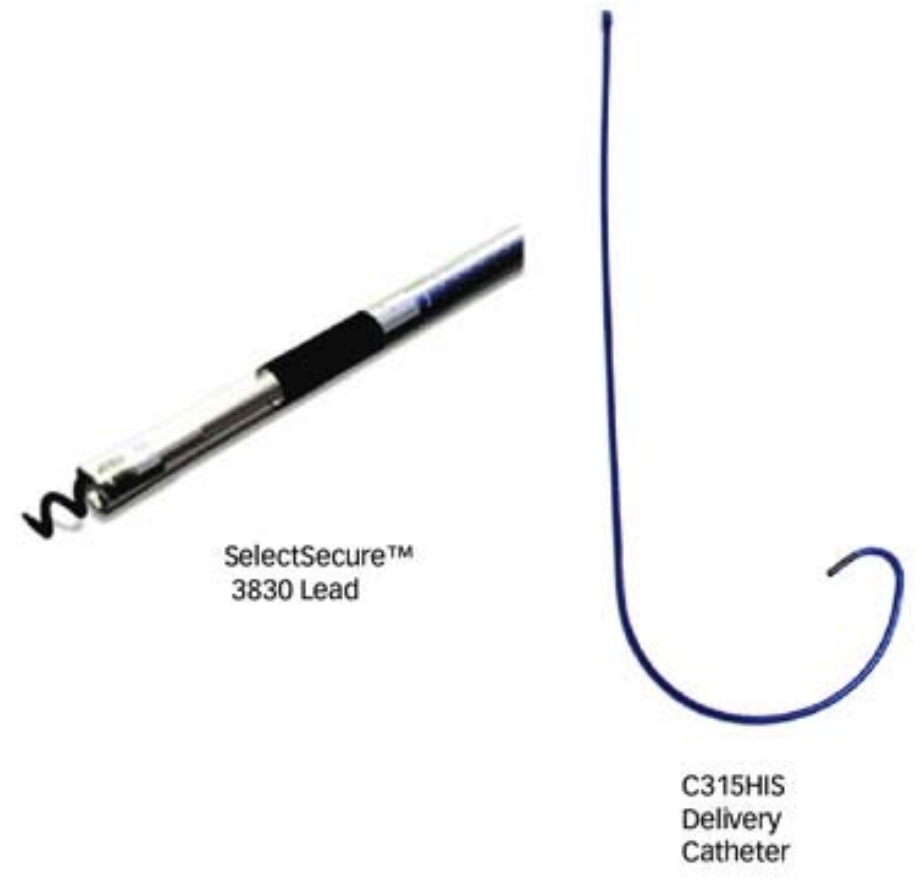

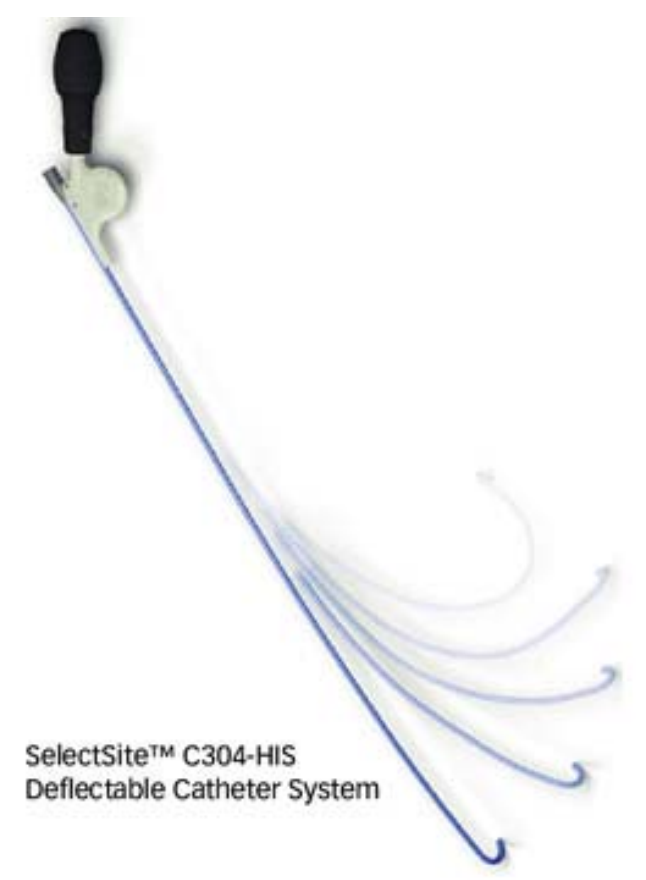

Figure I. Tools used for left bundle branch lead implant. 
tial effective refractory periods of the two structures $^{12}$. Premature beats are delivered either during sinus rhythm or after an 8-beat drive train at $600 \mathrm{~ms}$ with a $450 \mathrm{~ms}$ coupling interval decremented by 10 ms. Non-selective LBB pacing was characterized by paced QRS morphology changes to a myocardial-only capture (broader QRS, with slur/notch/plateau and/ or with change in amplitude/polarity in several leads). Selective LBBP was characterized by changed in QRS morphology to a typical RBB morphology preceded by a latency.

Once LBBP capture is recorded (narrow QRS with RBB morphology, higher R-wave amplitudes, short left ventricular activation time (LVAT) $\leq 60-90 \mathrm{msec}$, low capture threshold) no further lead advancement should be performed. A low capture threshold and high pacing impedance suggest that the pacing lead is located inside the septum. Sudden decrease of lead impedance, sensed R-wave amplitude, and/or loss of capture indicate that the helix of the lead has entered the chamber of the left ventricle, for example, LV perforation $^{8-10}$. Other potential complications that may occur during procedure are RBB injury, septal hematoma, coronary arterial injury, tricuspid regurgitation, risk of lead extraction.

Figure $2 \mathrm{C}$, D illustrates LBBP pacing in a patient with history of heart failure with low LVEF who had LBBB with QRSd of I 46 ms that was corrected with LBBP to QRSd of $110 \mathrm{~ms}$.
Left bundle branch pacing: the new kid on the block

\section{CLINICAL RESULTS}

In 2000 Deshmukh et al. conducted the pioneering investigation of permanent HBP in a small number of patients with dilated cardiomyopathy and chronic atrial fibrillation and reported this technique as a safe and effective physiological pacing method with improvements in cardiac function'. Since then a number of clinical studies have been conducted in patients with various cardiac disease to validate the feasibility, safety and effectiveness of the HBP method. Barba-Pichardo et al. ${ }^{2}$, Lustgarten et al. ${ }^{3}$ demonstrated that HBP provided clinical benefits and equivalent CRT response comparable to those of CRT via BIV.

Although HBP represent the most physiologic form of pacing, it is technically challenging due to anatomic location, long fluoroscopic exposure times, high capture thresholds, sensing issues and often fails to provide adequate pacing in patients with infrahisian block or proximal left bundle branch block. According to Upadhyay et al. ${ }^{13}$ the site of block usually is located within the His or proximal left bundle. Left bundle branch pacing overcomes the limitations of HBP by implanting the lead distal to the level of block ${ }^{5}$.

Studies of LBBP have focused on several issues: methods to improve technique feasability, safety, hemodynamic advantages, and pacing in common scenarios: bradycardia, complete or advanced degree $\mathrm{AV}$ block, atrial fibrillation with AV node ablation, and as an alternative to biventricular pacing for CRT in patients with heart failure.
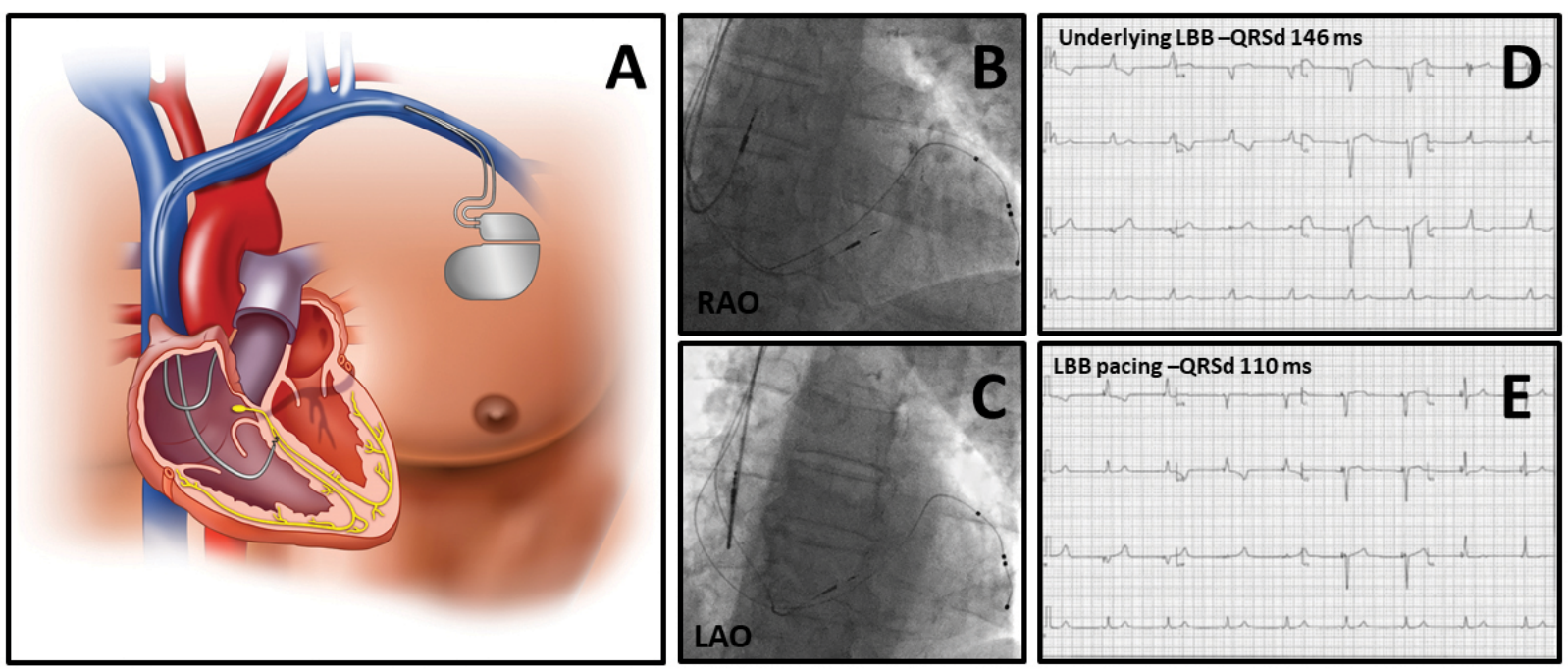

Figure 2. Example of left bundle branch implant in a patient with cardiac resynchronization therapy pacemaker.A. Typical location of left bundle lead position. B. RAO view showing location of RA, LB, LV leads. C. LAO view showing location of RA, LB, LV leads. D. Underlying rhythm - LBBB with QRSd I46 ms. E. LB paced rhythm with QRSd 110 ms. RAO - right anterior oblique; LAO - left anterior oblique; RA - right atrial; LB - left bundle; LV - left ventricular; LBBB - left bundle branch block; QRSd - QRS duration; ms - millisecond. 
Left bundle branch pacing is performed mostly using the 3830 lead and C 315 sheath. ${ }^{5}$ Mafi-Rad et al. ${ }^{14}$ developed another technique using a custom-made lead implanted in mid-distal septum. They observed that left ventricular septal pacing resulted in a narrower QRS with a RBB morphology and compared with RV pacing provided better hemodynamic benefits. 3D mapping guidence can be used to guide lead position and depth but it adds costs to the procedure ${ }^{15}$. The accurate location for LBBP can be targeted during implantation using characteristic changes in paced QRS morphology during lead deployment ${ }^{16}$. Similarly, Chen et al. ${ }^{10}$ in a prospective study explored the feasibility of LBBP using a transventricular septal approach and demonstrated that the transition of ECG morphology during lead implantation can guide the appropriate lead placement in the basal septum. At 3-months follow-up the pacing thresholds were comparable and remained low and stable. LBBP generated a narrower QRS duration (QRSd) due to fast left atrial activation and produced a better synchronization of the left ventricle than conventional $R V$ pacing.

LBBP preserves satisfactory LV synchrony and result in favorable hemodynamic effects in patients with narrow QRS when evaluated by echocardiography ${ }^{17}$. LBBP has beneficial effects in re-establishing ventricular electric and mechanical synchrony and LV reverse remodelling in patients with heart failure and $\mathrm{LBBB}^{18}$. In a single centre prospective study, using single-photon emission CT (SPECT) myocardial perfusion imaging, Hou et al. ${ }^{19}$ reported that mechanical synchrony parameters after LBBP were similar to HBP and better than RVP.

LBBP appears to be safe and feasible. In a two center study involving 34I patients, there were only 3 complications reported: all lead dislodgements ${ }^{20}$. LBBP was achieved in $89 \%$ of patients. In a long-term followup prospective study Vijayaraman et al. ${ }^{21,22}$ reported that $\mathrm{His}$ bundle capture threshold and the generator replacement rate in HBP was higher than that in RVP ( $9 \%$ vs. 1\%). They also described a high success rate $(88 \%)$ in the correction of LBBB by LBBP, and found that LBBP it is feasible in a high percentage of patients with low thresholds during acute follow-up and may significantly increase the overall success of physiologic pacing.

$\mathrm{Li}$ and colleagues $^{16}$ tested the feasibility and safety of LBBP in patients with AV nodal disease. LBBP was successful in $90 \%$ of patients, with a stable low pacing output and narrow paced QRS duration. The
LV mechanical synchrony evaluated by a 3-months follow-up echocardiography, was preserved or improved by LBBP. Guo et al. reported a $89 \%$ success rate in a series of 164 patients and no complications ${ }^{23}$. LBBP was shown to be safe in a small cohort of patients with heart failure, atrial fibrillation, and AV node ablation. Improvement in LVEF and reduction in LV end-systolic volume were significantly higher than in a control group that received standard ICD implant and had AV node ablation and resulted in a decreased incidence of inappropriate shocks ${ }^{24}$.

LBBP appears to be a good alternative for CRT. Zhang et al. ${ }^{18}$ evaluated the value of LBBP in eleven patients with heart failure, low LVEF and LBBB. They showed that LBBP has the potential to be an effective alternative to cardiac resynchronization therapy, by correcting the LBBB, providing ventricular electric and mechanical synchrony with LV reverse remodelling and improving the NYHA functional class. In propensity matched small cohort of patients with heart failu$\mathrm{re}$, $\mathrm{Li}$ et al., found that resulted in greater reduction in the QRSd (58.0 vs. $12.5 \mathrm{~ms}, \mathrm{P}<0.00 \mathrm{I})$, a greater increase in LVEF (I5.6\% vs. $7.0 \%, P<0.00 \mathrm{I})$, and greater echocardiographic ( $88.9 \%$ vs. $66.7 \%, P=0.035)$ and super response $(44.4 \%$ vs. $16.7 \%, P=0.007)$ to biventricular pacing ${ }^{25}$.

\section{CONCLUSIONS}

LBBP represents an attractive option for physiologic pacing in patients with conduction disease by providing a stable and low capture threshold. As it bypasses the level of block, LBBP can potentially be a first line pacing method in patients with iatrogenic AV block due AV node ablation. LBBP appears to provide significant advantages in heart failure patients by achieving electrical and mechanical synchrony of the left ventricle. However, randomized prospective clinical trials are needed to establish the patients that would most likely benefit from this pacing strategy. Furthermore, studies are needed to asses the long-term effects of septal contractile stress with LBBP.

\section{Conflict of interest: none declared.}
Abbreviations list
$\mathrm{AV}=$ atrioventricular
$\mathrm{BIV}=$ biventricular pacing
CRT = cardiac resynchronization therapy
ECG = electrocardiographic
$\mathrm{HBP}=\mathrm{His}$ bundle pacing
$\mathrm{HF}=$ heart failure 


$$
\begin{aligned}
& \text { IVS = interventricular septum } \\
& \text { LBB = left bundle branch } \\
& \text { LBBB = left bundle branch block } \\
& \text { LBBP = left bundle branch pacing } \\
& \text { LV = left ventricular } \\
& \text { LVAT = left ventricular activation time } \\
& \text { LVEF = left ventricular ejection fraction } \\
& \text { QRSd = QRS duration } \\
& \text { RBB = right bundle branch } \\
& \text { RBBB = right bundle branch block } \\
& \text { RV = right ventricular } \\
& \text { RVP = right ventricular pacing } \\
& \text { SA = sinoatrial }
\end{aligned}
$$

\section{References}

I. Deshmukh P, Casavant DA, Romanyshyn M, Anderson K. Permanent, direct His-bundle pacing: a novel approach to cardiac pacing in patients with normal His-Purkinje activation. Circulation. 2000; I0I (8):869-877.

2. Barba-Pichardo R, Morina-Vazquez P, Fernandez-Gomez JM, Venegas-Gamero J, Herrera-Carranza M. Permanent His-bundle pacing: seeking physiological ventricular pacing. Europace : European pacing, arrhythmias, and cardiac electrophysiology : journal of the working groups on cardiac pacing, arrhythmias, and cardiac cellular electrophysiology of the European Society of Cardiology. 2010;12(4):527533.

3. Lustgarten DL, Crespo EM, Arkhipova-Jenkins I, et al. His-bundle pacing versus biventricular pacing in cardiac resynchronization therapy patients: A crossover design comparison. Heart rhythm : the official journal of the Heart Rhythm Society. 20I5; I2(7): I548-I557.

4. Vijayaraman P, Chung MK, Dandamudi G, et al. His Bundle Pacing. Journal of the American College of Cardiology. 2018;72(8):927-947.

5. Huang W, Su L, Wu S, et al. A Novel Pacing Strategy With Low and Stable Output: Pacing the Left Bundle Branch Immediately Beyond the Conduction Block. The Canadian journal of cardiology. 2017;33(I2): I736 el73I-I736 el733.

6. Gu M, Li H, Hu YR, Niu HX, Hua W. Cardiac resynchronization therapy using left ventricular septal pacing: An alternative to biventricular pacing? HeartRhythm Case Rep. 2019;5(8):426-429.

7. Huang $\mathrm{W}, \mathrm{Wu} S$, Vijayaraman $\mathrm{P}$, et al. Cardiac Resynchronization Therapy in Patients With Nonischemic Cardiomyopathy Using Left Bundle Branch Pacing. JACC Clin Electrophysiol. 2020;6(7):849-858.

8. Huang W, Chen X, Su L, Wu S, Xia X, Vijayaraman P. A beginner's guide to permanent left bundle branch pacing. Heart rhythm : the official journal of the Heart Rhythm Society. 2019;16(I2): I79I-I796.

9. Sundaram S, Vijayaraman P. Left bundle branch pacing. Herzschrittmacherther Elektrophysiol. 2020;3 I (2): I24-I34.

10. Chen K, Li Y, Dai Y, et al. Comparison of electrocardiogram characteristics and pacing parameters between left bundle branch pacing and right ventricular pacing in patients receiving pacemaker therapy. Europace : European pacing, arrhythmias, and cardiac electrophysiology : journal of the working groups on cardiac pacing, arrhythmias, and cardiac cellular electrophysiology of the European Society of Cardiology. 2019;2I(4):673-680.
II. Chen K, Li Y. How to implant left bundle branch pacing lead in routine clinical practice. Journal of cardiovascular electrophysiology. 2019;30(I I):2569-2577.

12. Jastrzebski M, Moskal P, Bednarek A, et al. Programmed deep septal stimulation: A novel maneuver for the diagnosis of left bundle branch capture during permanent pacing. Journal of cardiovascular electrophysiology. 2020;3I(2):485-493.

13. Upadhyay GA, Cherian T, Shatz DY, et al. Intracardiac Delineation of Septal Conduction in Left Bundle-Branch Block Patterns. Circulation. 2019;139(16):1876-1888.

14. Mafi-Rad M, Luermans JG, Blaauw Y, et al. Feasibility and Acute Hemodynamic Effect of Left Ventricular Septal Pacing by Transvenous Approach Through the Interventricular Septum. Circulation Arrhythmia and electrophysiology. 2016;9(3):e003344.

15. Vijayaraman P, Panikkath R, Mascarenhas V, Bauch TD. Left bundle branch pacing utilizing three dimensional mapping. Journal of cardiovascular electrophysiology. 2019;30(I2):3050-3056.

16. Li Y, Chen K, Dai Y, et al. Left bundle branch pacing for symptomatic bradycardia: Implant success rate, safety, and pacing characteristics. Heart rhythm : the official journal of the Heart Rhythm Society. 2019;16(12):1758-1765.

17. Cai B, Huang $X$, Li L, et al. Evaluation of cardiac synchrony in left bundle branch pacing: Insights from echocardiographic research. Journal of cardiovascular electrophysiology. 2020;3 I(2):560-569.

18. Zhang W, Huang J, Qi Y, et al. Cardiac resynchronization therapy by left bundle branch area pacing in patients with heart failure and left bundle branch block. Heart rhythm : the official journal of the Heart Rhythm Society. 2019;16(12): 1783-1790.

19. Hou X, Qian Z, Wang Y, et al. Feasibility and cardiac synchrony of permanent left bundle branch pacing through the interventricular septum. Europace : European pacing, arrhythmias, and cardiac electrophysiology : journal of the working groups on cardiac pacing, arrhythmias, and cardiac cellular electrophysiology of the European Society of Cardiology. 2019;2 I (I I):1694-I702.

20. Santosh K. Padala VMM, Maria Terricabras, Andrea Chiocchini, Aatish Garg, Jordana Kron, Richard Shepard, Gautham Kalahasty, Zahara Azizi, Bernice Tsang, Yaariv Khaykin, Alfredo Pantano, Jayanthi N. Koneru, Kenneth A. Ellenbogen and Atul Verma. Initial Experience, Safety, and Feasibility of Left Bundle Branch Area Pacing: A Multicenter Prospective Study. JACC: Clinical Electrophysiology. 2020.

21. Vijayaraman P. His-bundle Pacing to Left Bundle Branch Pacing: Evolution of His-Purkinje Conduction System Pacing. J Innov Card Rhythm Manag. 2019;10(5):3668-3673.

22. Vijayaraman P, Subzposh FA, Naperkowski A, et al. Prospective evaluation of feasibility and electrophysiologic and echocardiographic characteristics of left bundle branch area pacing. Heart rhythm: the official journal of the Heart Rhythm Society. 2019; I6(I2): I774- 1782

23. Guo J, Li L, Meng F, et al. Short-term and intermediate-term performance and safety of left bundle branch pacing. Journal of cardiovascular electrophysiology. 2020;3 I(6): |472-I48I.

24. Wang S, Wu S, Xu L, et al. Feasibility and Efficacy of His Bundle Pacing or Left Bundle Pacing Combined With Atrioventricular Node Ablation in Patients With Persistent Atrial Fibrillation and Implantable Cardioverter-Defibrillator Therapy. Journal of the American Heart Association. 2019;8(24):e014253.

25. Li X, Qiu C, Xie R, et al. Left bundle branch area pacing delivery of cardiac resynchronization therapy and comparison with biventricular pacing. ESC Heart Fail. 2020;7(4): I7| I-I 722. 